\title{
Production of (anti)deuterons in heavy-ion collisions at SPS energies
}

\section{V.I. Kolesnikov* for the NA49 Collaboration}

Joint Institute for Nuclear Research, Dubna, Russia

E-mail: Vadim.Kolesnikovecern.ch

In this paper NA49 results on the production of deuterons and anti-deuterons in semi-central $\mathrm{Pb}+\mathrm{Pb}$ collisions at $158 \mathrm{~A} \mathrm{GeV}$ are presented. Midrapidity transverse momentum spectra of $d(\bar{d})$ are analysed in several centrality bins. A combined analysis of (anti)deuterons and (anti)protons iss performed in the framework of the coalescence model.

XXII International Baldin Seminar on High Energy Physics Problems,

15-20 September 2014

JINR, Dubna, Russia

\footnotetext{
* Speaker.
} 


\section{Introduction}

Study of (anti)deuteron production in heavy-ion collisions is interesting for many reasons. Enhanced antimatter production in central nucleus-nucleus collisions relative to $p+p$ was proposed as an experimental signature for the deconfinement phase transition and formation of a new state of strongly interacting nuclear matter - the Quark-Gluon Plasma. [1, 2, 3]. However, in central Pb+Pb collisions at SPS energies baryon rich nuclear matter is created, so that an enhanced antibaryon production might be shadowed due to high annihilation rates in the medium. New experimental data on anti-nuclei production can shed light on the interplay between the production and annihilation processes in the fireball.

At the late stage of the fireball evolution, when secondary inelastic collisions cease, the observed clusters are formed from nucleons. In a simplified coalescence approach [4, 5], (anti)nucleon bound states are formed from (anti)nucleons which are close in momentum and configuration space. At SPS energies the diameter of the source created in central collisions is much larger than the deuteron size due to expansion of the reaction zone, and the cluster production process becomes sensitive to the details of the phase-space distribution of the constituents. In particular, a comparative analysis of the production of clusters made of nucleons and antinucleons might provide insight into the details of the fireball evolution and the nucleon phase-space distribution at kinetic freeze-out. For example, if the $\bar{p}$ suffer from annihilation in the dense baryonic matter, this could therefore be reflected in the impact parameter dependence of the $\bar{d}$ yield [6].

\section{NA49 experiment and data analysis}

The NA49 apparatus is a large acceptance magnetic spectrometer covering about $50 \%$ of the final state phase space for $\mathrm{Pb}+\mathrm{Pb}$ reactions at $\mathrm{SPS}$ energies. The detector provides precise tracking and efficient particle identification with four Time-Projection Chambers (TPCs) and two scintillator Time-Of-Flight (TOF) walls, complemented by event centrality measurements in a Zero Degree Calorimeter (ZDC). A full description of the apparatus components can be found in Ref. [7].

For this study, an event sample of $2.4 \cdot 10^{6}$ events recorded during the year 2000 run period with an online event trigger selecting the $23.5 \%$ most central collisions was used. To study the centrality dependence of the $\bar{d}$ and $d$ yields the data set was divided off-line into two centrality classes: $0-12 \%$ and $12-23 \%$ central.

A set of event selection criteria and track quality cuts was applied in the analysis procedure. An event should have a reconstructed vertex and the difference between its $z$-position and the nominal target position was required to be less than $1 \mathrm{~cm}$. Track candidates are required to have a valid momentum fit result and $d E / d x$ measurement in the TPC, and for each track both vertical and horizontal deviations from the vertex position, after back extrapolation to the $z$ position of the target, were required to be less than $1 \mathrm{~cm}$. In order to reject those TOF hits that have poorly reconstructed time of flight, all candidates having two or more tracks in the event hitting the same scintillator were rejected, In addition, a cut on the energy deposited in a TOF scintillator was applied to reduce background under the (anti)deuteron mass peak due to false TOF hits and eventcorrelated $\gamma$-interactions. The fraction of the tracks remaining after all the quality cuts was found to be $67 \%$. 


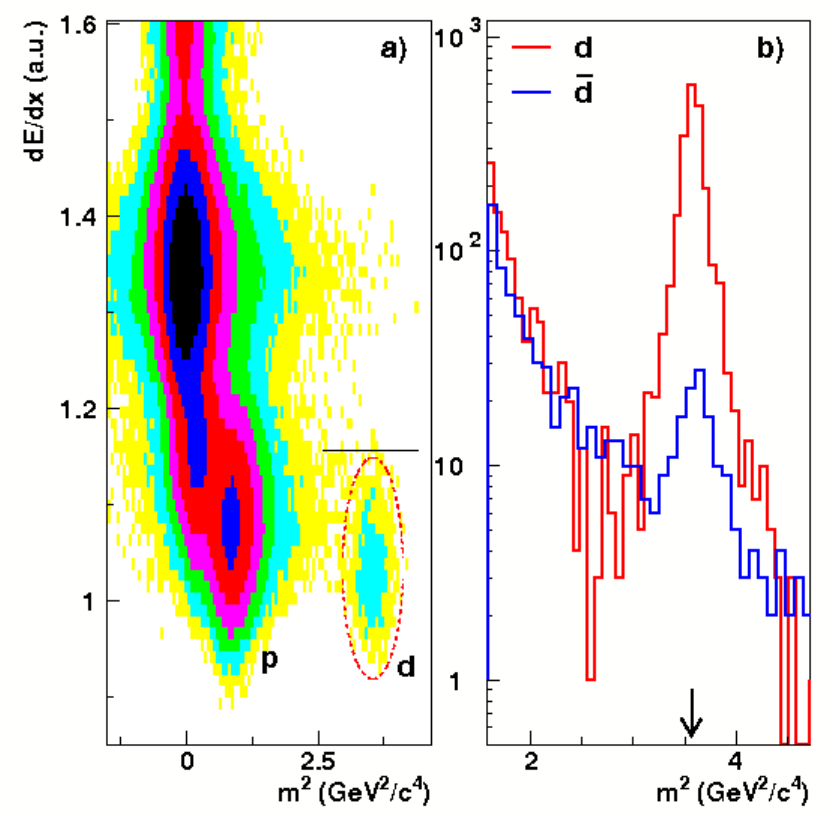

Figure 1: a) $d E / d x$ versus $m^{2}$ for positively charged particles in the momentum interval $7<p<8 \mathrm{GeV} / c$. The dashed ellipse illustrates the region populated by deuterons; the PID upper-limit $d E / d x$ cut is indicated by the horizontal line. b) $m^{2}$-distributions for both charges around the $m_{d}^{2}$ position for momenta from 4 to $10 \mathrm{GeV} / c\left(d E / d x\right.$ PID cut for $d(\bar{d})$ was applied). The arrow indicates the nominal $m^{2}$ position for $d(\bar{d})$.

The particle identification is done by combining measurements of time-of-flight from the TOF detector and $d E / d x$ from the MTPCs (Fig. 1, a). In order to reduce the contribution of pions and kaons in (anti)nuclei, an upper-limit $d E / d x$-cut was applied based on a momentum dependent parameterization of the Bethe-Bloch curve for deuterons. The resulting $\mathrm{m}^{2}$-distributions for positively and negatively charged particles after applying the previously described quality and $d E / d x$ PID cuts show clear $d$ and $\bar{d}$ peaks (Fig. 1, b). The entire track sample of $\bar{d}(d)$ was divided into several transverse momentum bins and in each bin candidates are selected by a $\pm 3 \sigma$ window in $m^{2}$ around the nominal position. The raw yields were determined by summing the histogram bin contents within the mass window. The background contamination under the $\bar{d}(d)$ peak was evaluated by fitting the $m^{2}$-distribution to a sum of a Gaussian signal and an exponential background. The raw yields were weighted by correction factors which account for the geometrical acceptance and for the losses due to the applied cuts. The systematic errors of the $d$ and $\bar{d}$ yields originate mainly from the uncertainties of particle identification and efficiency correction factors. Each particular contribution to the overall systematic error was estimated by varying the characteristic selection criteria. The overall systematic error, obtained as the quadratic sum of all contributions, was estimated to be $17 \%$ for $\bar{d}$ and $10 \%$ for $d$. More details about the analysis procedure can be found in [8]. 


\section{Results}

\subsection{Transverse momentum spectra of $d(\bar{d})$ and $p(\bar{p})$}

In Fig. 2(a,b) the invariant $p_{t}$ spectra of $d$ and $\bar{d}$ for centrality selected $\mathrm{Pb}+\mathrm{Pb}$ collisions are plotted as a function of transverse momentum $p_{t}$. The yields are averaged over the center-of-mass rapidity interval $-1.2<y<-0.6$. The spectra for $p$ and $\bar{p}$ in the same centrality selected event samples are also shown in Fig.2(c,d). The experimental distributions are fitted to an exponential
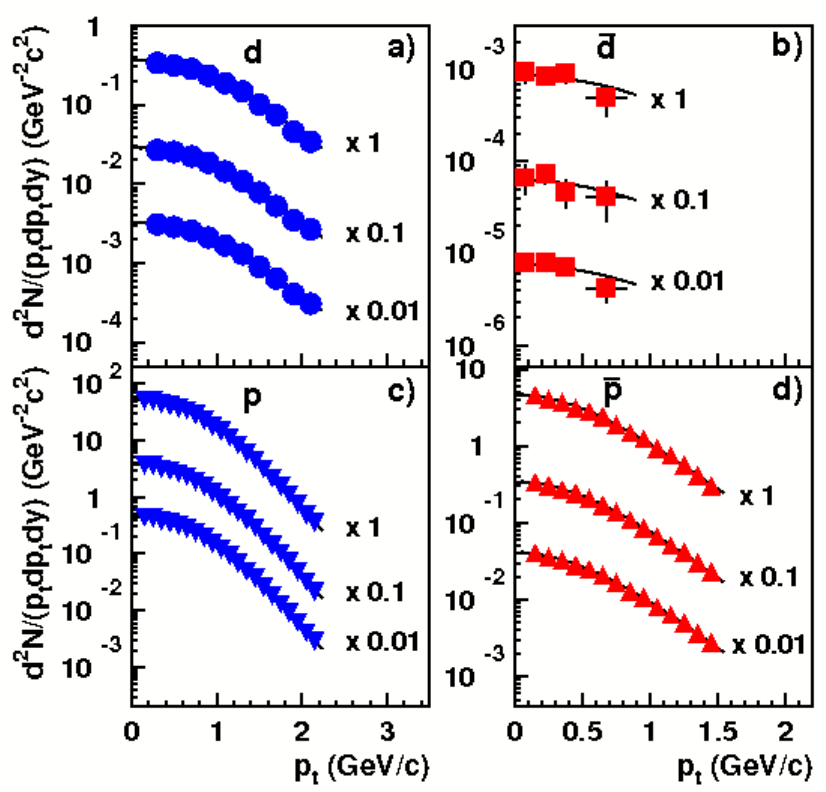

Figure 2: The transverse momentum spectra of deuterons (a), antideuterons (b), protons (c) and antiprotons (d) in centrality selected $\mathrm{Pb}+\mathrm{Pb}$ collisions at $158 \mathrm{~A} \mathrm{GeV}$. Only statistical errors are shown. The curves show exponential fits to the data. Results for the $0-12.5 \%$ most central collisions are shown to scale, results for the centrality intervals $12.5-23.5 \%$ and $0-23.5 \%$ are scaled down by factors 10 and $10^{2}$ respectively.

function in $m_{t}$ (shown by curves in Fig. 2)

$$
\frac{1}{p_{t}} \frac{d^{2} N}{d p_{t} d y}=\frac{d N / d y}{T(m+T)} \exp \left(-\frac{m_{t}-m}{T}\right)
$$

where $d N / d y$ and $T$ are two fit parameters, $m_{t}=\sqrt{p_{t}^{2}+m^{2}}$ is the transverse mass and $m$ the particle's rest mass.

The small available statistics and resulting limited $p_{t}$ range makes the determination of the slope parameters for $\bar{d}$ less accurate. Thus only the $d N / d y$ parameter was allowed to vary when the $\bar{d}$-spectra were fitted and merely a qualitative comparison of the shape of the spectra is possible. The slope parameter $T$ for deuterons is $406 \pm 12 \mathrm{MeV}$ and $391 \pm 12 \mathrm{MeV}$ for the $0-12.5 \%$ and $12.5-23.5 \%$ most central collisions, respectively. It tends to increase with increasing centrality indicating increase of collective transverse flow. The invariant $p_{t}$ spectra of $\bar{d}(d)$ are harder than 


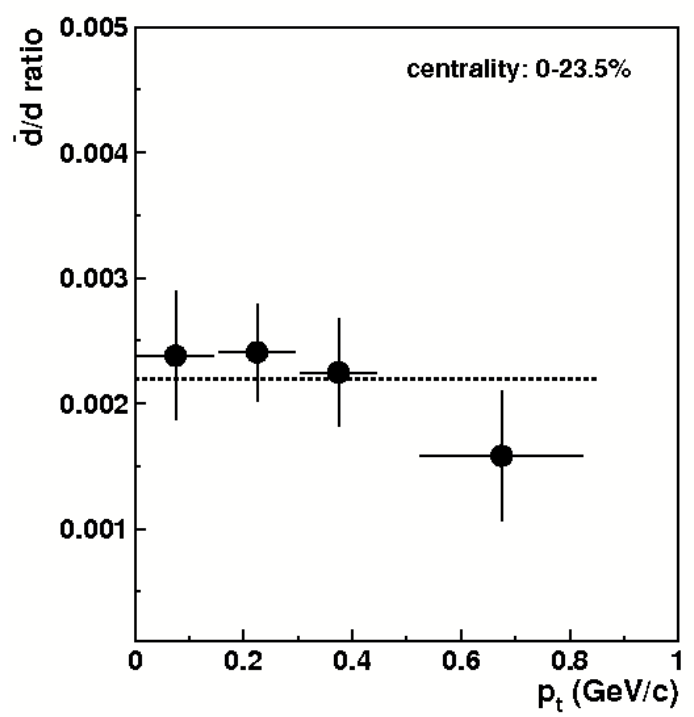

Figure 3: $\bar{d} / \mathrm{d}$-ratio as a function of $p_{t}$ in $0-23.5 \%$ central $\mathrm{Pb}+\mathrm{Pb}$ collisions at $158 \mathrm{~A} \mathrm{GeV}$. The dashed line shows a fit to a constant.

those of $\bar{p}(p)$ (typical $T_{p} \approx 290-300 \mathrm{MeV}$ ). The hardening of the $p_{t}$-spectra with the particle mass supports a box-like density profile in the expanding fireball, as suggested in $[9,10]$.

Calculations using the microscopic transport model UrQMD [11] suggest that, if annihilation of antimatter in the fireball medium affects $\bar{d}$ production, then one would expect $\bar{d}$ losses to be larger at low $p_{t}$ [12], resulting in a hardening of the midrapidity $p_{t}$ spectra of $\bar{d}$ compared to those for $d$. Fig. 3 shows the $\bar{d} / d$ ratio for the $0-23 \%$ centrality bin as a function of $p_{t}$. Since a little variation with $p_{t}$ is observed and there is no indication of extra reduction of the $\bar{d}$ yield at low $p_{t}$, a likely conclusion is that the dynamical properties of the formation process are the same for $d$ and $\bar{d}$. A fit with a constant yields an overall $\bar{d} / d$ ratio of $(2.2 \pm 0.2) \cdot 10^{-3}$.

\subsection{Centrality dependence of $\bar{d}$ and $d$ yields}

The measured invariant yield of $\bar{d}$ and $d$ per a wounded nucleon is shown in Fig. 4 as a function of $\left\langle N_{w}\right\rangle$. The data from this study are shown together with the previously published NA49 results for deuterons from the year 1996 minimum bias data set [13] (shown as blue circles). The observed centrality dependence of the $\bar{d}$ yield per $N_{w}$ appears to be weak in mid-central $\mathrm{Pb}+\mathrm{Pb}$ collisions. The same is true for the normalized yield of $d$ showing little variation in the centrality range under study (except a jump of about $15 \%$ at $\left\langle N_{w}\right\rangle \approx 180$ ). This behavior can be understood as an indication of some degree of saturation in the density distribution of both nucleons and antinucleons achieved in mid-central $\mathrm{Pb}+\mathrm{Pb}$ collisions at the top SPS energy.

\subsection{Rapidity distributions and total yields of $d$ and $\bar{d}$}

Yields of $d$ and $\bar{d}$ in rapidity slices from the $23.5 \%$ central $\mathrm{Pb}+\mathrm{Pb}$ collisions are presented in Fig. 5. The shapes of the rapidity distributions for $d$ and $\bar{d}$ differ drastically: the rapidity distribution for deuterons has a dip at midrapidity in contrast to the peak observed for antideuterons. In Fig. 5 


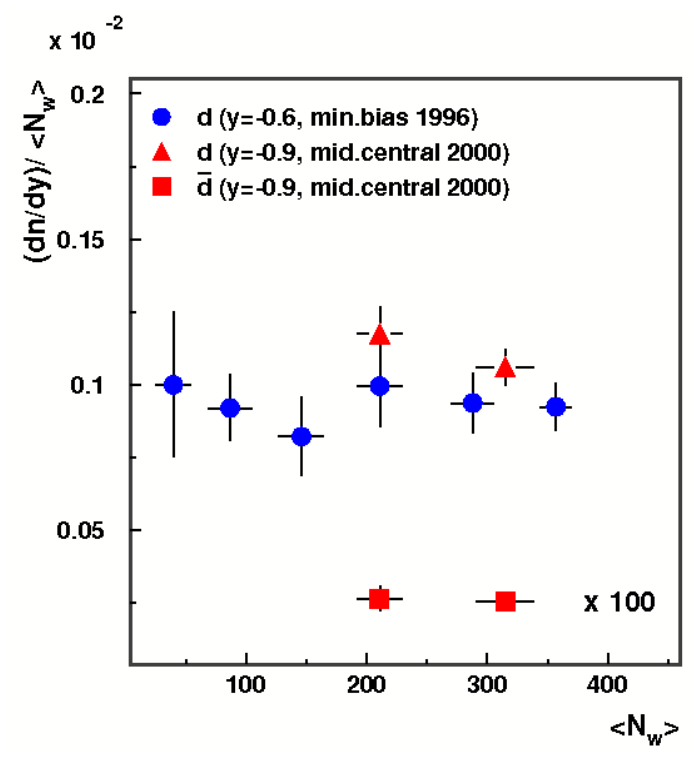

Figure 4: $d N / d y$ divided by the average number of wounded nucleons $\left\langle N_{w}\right\rangle$ for $\bar{d}$ and $d$ from $\mathrm{Pb}+\mathrm{Pb}$ collisions at $158 \mathrm{~A} \mathrm{GeV}$.

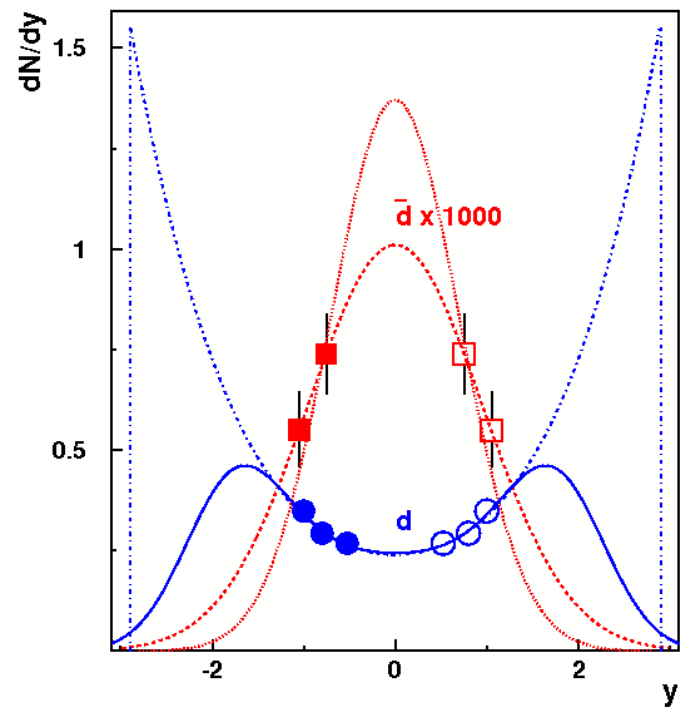

Figure 5: Rapidity distributions for $\bar{d}$ (squares) and $d$ (circles) produced in the $23.5 \%$ most central $\mathrm{Pb}+\mathrm{Pb}$ collisions at $158 \mathrm{~A} \mathrm{GeV}$. The solid symbols indicate the measured data points, the open symbols are reflected at midrapidity. The curves represent the results of different fits motivated by a coalescence approach (see text). 


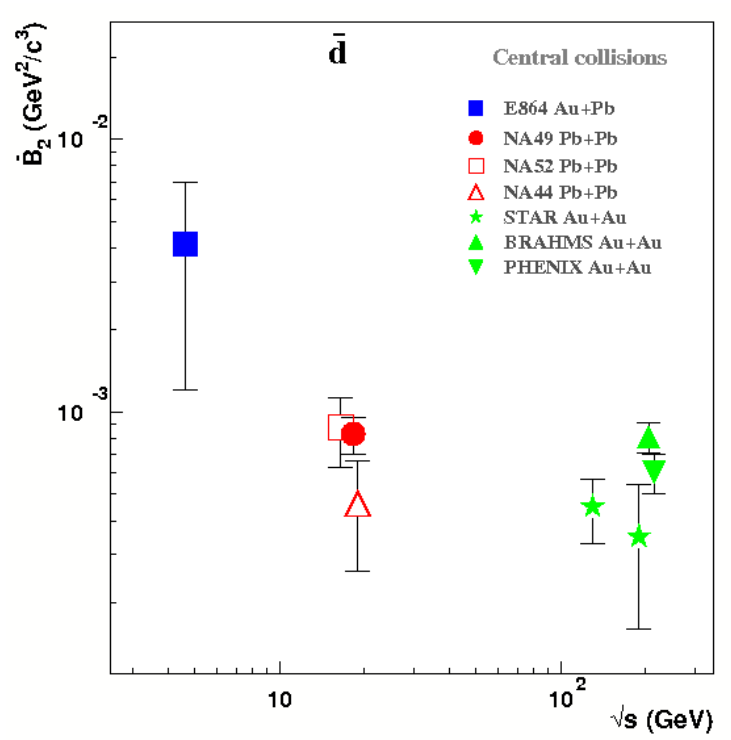

Figure 6: Coalescence parameters $B_{2}$ for $\bar{d}$ in central A+A interactions at different collision energies.

two normal distributions are plotted with the dotted line representing a Gaussian with coalescence motivated parameters based on the experimental data on antiproton production from [14]. The integral of this distribution is equal to that of the second Gaussian (shown by dashed line), whose parameters, i.e. the width and total yield were calculated based on the measured points. The calculated parameters were found to be $2.4 \cdot 10^{-3}$ and 0.95 for the $4 \pi$-yield and for the width. The width of the rapidity distribution for anti-deuterons is close to that observed for antiprotons $\left(\sigma_{\bar{p}} \approx 0.93-1.03\right)$ in the centrality range under study [14].

In the case of the rapidity distribution for $d$ our estimates for the total yield based on parametrizations to the proton spectrum [14] in the region covered by NA49 data. We used two alternative shapes: a parabolic one (see a dash-dotted curve in Fig. 5) and a sum of three Gaussians (shown by a solid line in Fig. 5). Both shapes fit the data points well and result in an estimate of the total $d$ yield of $2.8 \pm 0.28$ and $1.75 \pm 0.18$ for the parabolic and Gaussian shape, respectively.

\subsection{Coalescence}

A combined analysis of the spectra of light (anti)nuclei and (anti)nucleons in the framework of a coalescence approach $[4,5]$ relates the invariant yield of $Z$-charged $A$-clusters ( $A$ denotes the atomic mass number) to the product of the yields of protons $(p)$ and neutrons $(n)$ as

$$
E_{A} \frac{d^{3} N_{A}}{d^{3} P}=B_{A}\left(E_{p} \frac{d^{3} N_{p}}{d^{3} p}\right)^{Z}\left(E_{n} \frac{d^{3} N_{n}}{d^{3} p}\right)^{A-Z}
$$

The (unmeasured) yield of neutrons is usually considered to be equal to that of protons. Thermal models of cluster production $[15,16]$ predict the $B_{A}$ coefficient (coalescence parameter) to be inversely proportional to the volume of the particle source. Our measurement of $B_{2}$ along with other recent experimental results for the coalescence parameter for antideuterons [17, 18, 19, 20, 21] is shown in Fig. 6. The experimental data indicate that there is no substantial decrease of the 
coalescence parameter in the region of $\sqrt{s}$ from 17 to $200 \mathrm{GeV}$. This implies that the transverse size of the emitting source for $\bar{d}$ at kinetic freeze-out depends only weakly on the collision energy in this energy domain.

\section{Summary}

In this report recent results on $\bar{d}$ and $d$ production in the $23.5 \%$ most central $\mathrm{Pb}+\mathrm{Pb}$ collisions at $158 \mathrm{~A} \mathrm{GeV}$ from the NA49 experiment are presented. Anti-deuterons are measured in the transverse momentum interval $0<p_{t}<0.9 \mathrm{GeV} / c$. A comparison of the $p_{t}$ distributions for $\bar{d}$ and $d$ showed close similarity of spectral shapes. Moreover, the rapidity density of (anti)nuclei normalized to the number of wounded nucleons exhibits no centrality dependence. Thus no obvious effects of annihilation in the fireball medium were observed. A strong difference was observed in the shapes of the rapidity distributions for $\bar{d}$ (convex) and $d$ (concave). The total yields were estimated from a Gaussian and parabolic fit for $\bar{d}$ and $d$, respectively.

\section{References}

[1] U. Heinz et al., J. Phys. G 12 (1986) 1237.

[2] P. Koch et al., Mod. Phys. Lett. A 3 (1988) 737.

[3] J. Ellis et al., Phys. Lett. B 233 (1989) 223.

[4] S. T. Butler and C. A. Pearson, Phys. Rev. 129 (1963) 836.

[5] A. Schwarzschild and C. Zupancic, Phys. Rev. 129 (1963) 854.

[6] M. Bleicher et al., Phys. Lett. B 361 (1995) 10.

[7] S. V. Afanasiev et al (NA49 Collaboration), Nucl. Instrum. Meth. A430 (1999) 210.

[8] T. Anticic et al. (NA49 Collaboration), Phys. Rev. C 85 (2012) 044913.

[9] A. Polleri et al., Phys. Lett. B 419 (1998) 19.

[10] R. Scheibl and U. Heinz, Phys. Rev. C 59 (1999) 1585.

[11] M. Bleicher et al., J. Phys. G 25 (1999) 1859.

[12] F. Wang, J. Phys. G 27 (2001) 283.

[13] T. Anticic et al. (NA49 Collaboration), Phys. Rev. C 69 (2004) 024902.

[14] T. Anticic et al. (NA49 Collaboration), Phys. Rev. C 83 (2011) 014901.

[15] J. Kapusta, Phys. Rev. C 21 (1980) 1301.

[16] A. Z. Mekjian, Phys. Rev. C 17 (1978) 1051.

[17] T. A. Armstrong et al. (E864 Collaboration), Phys. Rev. Lett. 85 (2000) 2685.

[18] I. G. Bearden et al. (NA44 Collaboration), Phys. Rev. Lett. 85 (2000) 2681.

[19] C. Adler et al. (STAR Collaboration), Phys. Rev. Lett. 87 (2001) 262301.

[20] S.S. Adler et al. (PHENIX Collaboration), Phys. Rev. Lett. 94 (2005) 122302.

[21] I. Arsene et al. (BRAHMS Collaboration), preprint [nucl-ex] 1005.5427 (2010). 